\title{
A USABILIDADE DO APLICATIVO WHATSAPP ENQUANTO RECURSO DE APOIO AS MEDIAÇÕES DIDÁTICAS A DISCIPLINA REMOTA DE TCC NO CURSO DE ENGENHARIA CIVIL E DE PRODUÇÃO
}

\section{DOI: 10.37702/2175-957X.COBENGE.2021.3600}

Ana DArc Maia Pinto - ana.darc@terra.com.br

Centro Universitário Anhanguera de Niterói

Avenida do Rio Branco 123

24020-000 - Rio de Janeiro - RJ

Karoline Brito Coutinho Ferreira - karolinebritocoutinho.ferreira@gmail.com

Centro Universitário Anhanguera de Niterói

Rua Doutor Alfredo Backer 458

24452-005 - São Gonçalo - RJ

Resumo: No cenário atual, com a suspensão das aulas presenciais os meios digitais tornaram-se imprescindíveis. Dessa forma, o presente estudo tem como objetivo promover uma investigação da usabilidade do aplicativo WhatsApp enquanto recurso de apoio as mediações didáticas as disciplinas remotas no curso de engenharia civil e de produção em uma instituição privada de ensino superior. $E$ ́ notório constatar o uso expansionista dos aplicativos (APPS) como um recurso funcional para subsidiar diferentes serviços. Essa funcionalidade tornou-se possível pelo progresso do ciberespaço, associado ao advento dos smartphones, que possuem, entre suas características, a interconectividade e sua a fácil utilização. Movido por este panorama, este estudo buscou como trilha metodológica combinar a pesquisa quantitativa a qualitativa com um viés descritivo. Assim, seus resultados evidenciaram o quanto o aplicativo em voga alinha-se a possibilidades robustas enquanto recurso de apoio as mediações didáticas as disciplinas remotas dos Cursos em questão.

Palavras-chave: Aplicativos de Comunicação, Práticas Didáticas Colaborativas e Estudantes de Engenharia. 


\section{A USABILIDADE DO APLICATIVO WHATSAPP ENQUANTO RECURSO DE APOIO AS MEDIAÇÕES DIDÁTICAS A DISCIPLINA REMOTA DE TCC NO CURSO DE ENGENHARIA CIVIL E DE PRODUÇÃO}

\section{INTRODUÇÃO}

As mudanças pelas quais o contexto universitário hoje passa exige transformações em relação à docência nas Instituições de Ensino Superior. Esta constatação faz emergir a necessidade de apresentar alternativas que superem o mero utilitarismo do saber. No campo do ensino superior, Morés (2017) revela a partir de seus estudos que, as recentes transformações neste sistema de ensino exigem reorientação e reorganização profundas para que a universidade possa responder de modo criativo e eficaz aos novos desafios. É associado a este pressuposto que os aplicativos de comunicação se mostram como um recurso favorável para sustentação de práticas didáticas colaborativas.

Para Costa et al (2017) os aplicativos abriram muitas possibilidades para abordagens diferenciadas na educação, isso porque estes recursos tecnológicos carregam em suas interfaces atributos comunicacionais que ultrapassam as barreiras de tempo e do espaço. Alinhado a esta premissa, estudos de Saccol, Schlemmer e Barbosa (2011) destacam que alguns aplicativos de comunicação utilizados enquanto recursos pedagógicos podem instigar as habilidades cognitivas dos alunos e, acima de tudo, proporcionar situações para que possam utilizar seus novos conhecimentos para a solução de problemas. Já na perspectiva dos atributos, a norma ISO/IEC 9126 define algumas características que um aplicativo de comunicação deve apresentar como: confiabilidade de desempenho; eficiência no que tange aos recursos; funcionalidade; manutenibilidade; portabilidade (possibilidade de uso e coexistência em diversas plataformas e sistemas operacionais) e por fim, a usabilidade.

Ainda, Costa et al (2017) ressalta em seus estudos que estes atributos tornam os aplicativos "espaços" vigorosas para as trocas, proporcionando aos professores e estudantes mobilidade e uma interface fácil de ser utilizada, podendo assim, implementar diferentes estratégias de ensino e aprendizagem.

Briz-Ponce, Juanes-Mendez, Garcia-Penalvo e Pereira (2016), realizaram um estudo com o objetivo de conhecer quais fatores podem influenciar o comportamento de estudantes universitários que se utilizaram de aplicativos comunicacionais enquanto recurso tecnológico de sustentação dos processos pedagógicos. Os achados desse estudo deixaram evidentes que a influência social é um fator preponderante para a atitude de intenção comportamental do uso da aprendizagem móvel. Buscando ampliar ainda mais essa discussão, Figueiredo, Godejord e Rodrigues (2016), realizaram um estudo cujo com o objetivo foi o de desenvolver um aplicativo direcionado para smartphones e tablets, com intuito de auxiliar as atividades educacionais na sala de aula. Os resultados dessa pesquisa indicaram que o aplicativo utilizado contribuiu positivamente para expansão da sala de aula além do ambiente fisico. Al-Emran, Elsherif e Shaalan (2016), realizaram uma pesquisa com o propósito de explorar quais as variáveis que inflenciam 0 uso do m-learning (aprendizagem móvel) em universidades localizadas no Oriente Médio para os discentes e docentes. Os resultados indicaram que os fatores sexo, idade e país contribuíram para incentivar o uso da aprendizagem móvel via smartphone na sala de aula. Já estudos de Vázquez-Cano (2014) cujo objetivo geral se concentrou em avaliar o uso didático de um aplicativo que foi criado especificamente para ajudar na melhoria da aprendizagem dos alunos universitários na Europa revelaram em suas considerações finais que o uso desse aplicativo foi altamente valorizado pelos alunos, por que 
possibilitou conexões entre os sujeitos e facilitou a assimilação dos conteúdos repassados pelos professores.

Corroborando com os esforços de estudo sobre o objeto em voga, Adegbija e Bola (2015), investigaram a percepção de universitários sobre a adoção de tecnologias móveis (uso de aplicativos) na aquisição do conhecimento. Os resultados deste estudo apontaram que esse tipo de recurso tecnológico de comunicação influencia de forma consistente na construção intelectual dos alunos além de estimular as interações sociais do grupo em questão. Seow e Wong (2016) desenvolvem um aplicativo de jogos para dispositivos móveis com o objetivo incentivar os estudantes a aprenderem a contabilidade de uma forma divertida, fora da sala de aula. Os estudantes avaliaram positivamente 0 aplicativo como uma forma divertida e interessante de aprender as rotinas contábeis.

Alshraideh e Al-Shrida (2017), procuraram investigar os fatores que influenciam a adoção por parte dos estudantes universitários da Jordânia em relação à aprendizagem móvel, baseada na teoria unificada de aceitação e uso da tecnologia (UTAUT). As considerações finais deste estudo sugerem que há um impacto positivo e significativo da expectativa de desempenho, expectativa de esforço e influência social na intenção de atitude dos estudantes na adoção da aprendizagem móvel. Estes pressupostos teóricos consubstanciam de forma preliminar as possibilidades deste estudo, apontando seu potencial e vigor ao campo em discussão.

\section{O UNIVERSO DA PESQUISA E A METODOLOGIA}

Ciribell (2003) afirma que o método científico pode ser definido como um conjunto de etapas e instrumentos pelo qual o pesquisador científico, direciona seu estudo de pesquisa com critérios de caráter científico para alcançar dados que suportam ou não sua teoria inicial. Desta forma, para alcançar o objetivo proposto por este estudo, iniciou-se a formação de um grupo de apoio à disciplina de TCC oferecida aos cursos de engenharia civil e de produção em que uma das pesquisadoras deste estudo esteve responsável. Num segundo momento, depois do grupo de apoio formado e em comum acordo com os estudantes, buscou-se eleger entre os aplicativos de comunicação mais usuais um que pudesse ser utilizado como aditivo as interações pedagógicas a disciplina em questão que atualmente, em virtude da Covid 19, foi oferecida remotamente. Assim sendo, por maioria, elegeu-se o WhatsApp. Este caminho metodológico tomado inicialmente, se aproximou de um grupo focal. Godoy e Munari (2006) afirmam que as técnicas de grupo, com certo destaque para os grupos focais, têm sido exploradas em larga escala pelos pesquisadores para a coleta de dados. Este pressuposto é reforçado por estudos de Kitzinger (2000), ao relatar que o grupo focal é uma forma de solidificar uma estratégia de coleta de dados baseada na comunicação e na interação dos participantes. Seu principal objetivo é reunir informações detalhadas sobre um tópico específico (sugerido por um pesquisador, coordenador ou moderador do grupo) a partir de um grupo de participantes selecionados. Ele busca colher informações que possam proporcionar a compreensão de percepções, crenças, atitudes sobre um tema, produto ou serviços. Seguidamente a estes passos tomou-se como conduta para levantamento dos dados para este estudo trabalhar na confecção de um questionário. A construção de um questionário, segundo Aaker et al. (2001), e considerada uma "arte imperfeita", pois não existem procedimentos exatos que garantam que seus objetivos de medição sejam alcançados com boa qualidade. Frente ao desafio, buscou-se então empregar na formulação das perguntas questões que buscassem refletir de forma mais direta possível o objetivo desse estudo. Levando em conta este momento metodológico, a pesquisa se volta a um esforço misto entre as 
abordagens quantitativa e qualitativa para que a partir dos dados subsidiassem as descrições pertinentes a investigação.

Com este intuito, foram elaboradas questões de múltipla escolha que simplificassem o processo de aplicação e análise dos resultados, reduzissem erros e tomasse pouco tempo dos respondentes. Desta forma, o formulário proposto foi elaborado pela plataforma Google Forms, com 11 perguntas e foram disponibilizados pela ferramenta de comunicação WhatsApp, de forma não obrigatória, em um grupo amostral de 140 pessoas. Obtendo respostas de 52 estudantes dos cursos de engenharia, seguindo um padrão de respostas múltipla escolha. Em desdobramento ao procedimento, seguem descritas as perguntas que fizeram parte do questionário disponibilizados aos estudantes:

1. Qual sua faixa-etária?

2. Qual a sua satisfação com a utilização do WhatsApp?

3. Durante o curso de graduação, você utilizou o WhatsApp como apoio para as disciplinas?

4. Os grupos criados, foram eficazes para a sua aprovação na disciplina?

5. A utilização do WhatsApp influenciou na sua postura como aluno?

6. Você recebeu informações sobre trabalhos, datas de provas e outros informes pelos grupos?

7. A troca de informações no grupo contribuiu para solucionar dúvidas?

8. Os professores fazem ou faziam parte do grupo?

9. A presença do professor no grupo é:

10.0 WhatsApp, como ferramenta de educação, contribui para a relação professor/aluno?

11. Como você classifica a utilização do WhatsApp como ferramenta de educação?

Figura 1 - Exemplo de resposta no formulário de avaliação

1. Qual sua faixa-etária?

De 17 a 24 anos

De 25 a 34 anos

De 35 a 45 anos

De 46 a 54 anos

Mais de 55 anos

Fonte: Autores (2021).

Ao fim da pesquisa, com as respostas alcançadas, trabalhou-se na elaboração dos gráficos representativos de cada pergunta realizada. 


\section{RESULTADOS}

As análises e os gráficos foram gerados pelo Google Forms a partir das respostas que foram recebidas pelo formulário. Ressaltando que os respondentes desta pesquisa são estudantes dos cursos de engenharia civil de produção do Centro Universitário Anhanguera de Niterói. Conforme demonstrado na figura 2, a maior parte dos estudantes deste universo, estão na faixa etária de 17 a 24 anos.

Figura 2 - Faixa etária dos estudantes
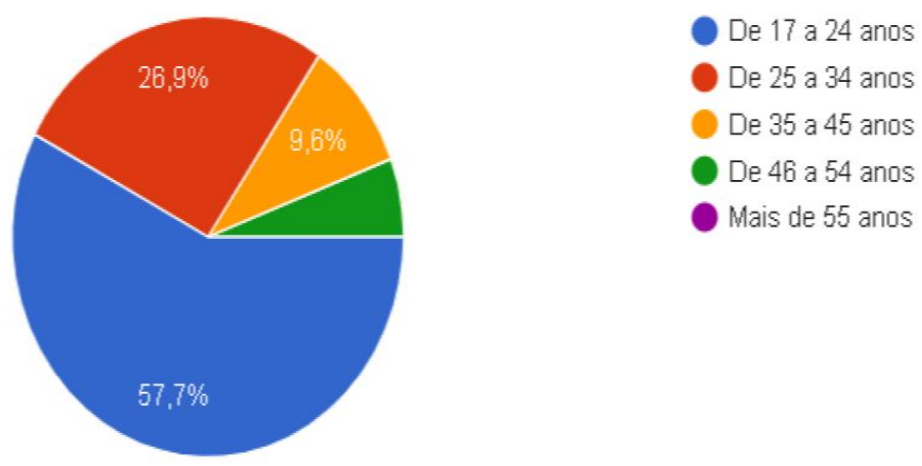

Fonte: Autores (2021).

Na segunda pergunta, demonstrada na figura 3 , os estudantes se demonstram apena satisfeitos com a ferramenta WhatsApp.

Figura 3 - Satisfação com a ferramenta utilizada

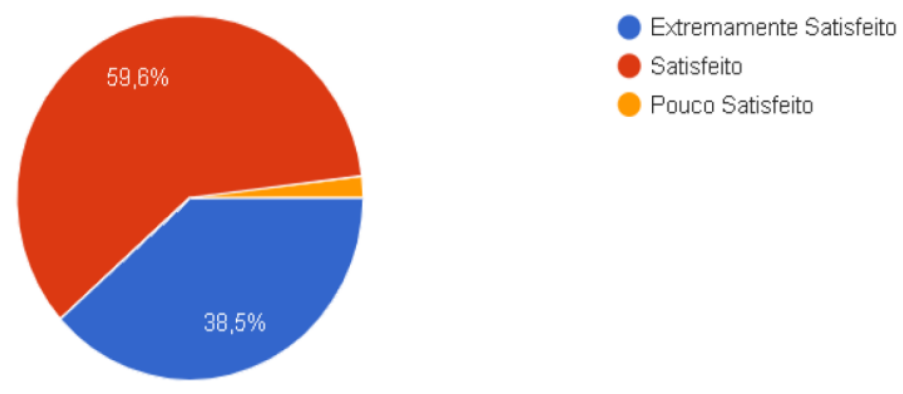

Fonte: Autores (2021).

$\mathrm{Na}$ pergunta 3,51 dos estudantes afirmaram que utilizaram o WhatsApp como apoio para as disciplinas dos cursos de engenharia, conforme demonstrado na figura 4 : 
Figura 4 - Utilização do WhatsApp como apoio nas disciplinas

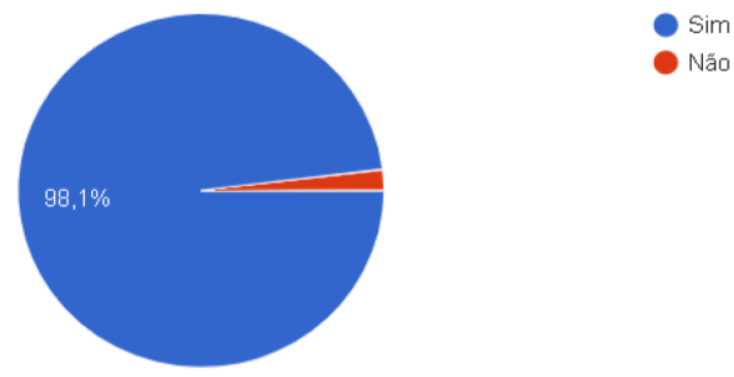

Fonte: Autores (2021).

Segundo os dados obtidos pelo gráfico, $69,2 \%$ dos estudantes demonstraram que os grupos de WhatsApp foram eficazes na sua aprovação para as disciplinas ministradas pelos cursos, conforme demonstrado na figura 5 :

Figura 5 - Utilização da ferramenta como item para aprovação

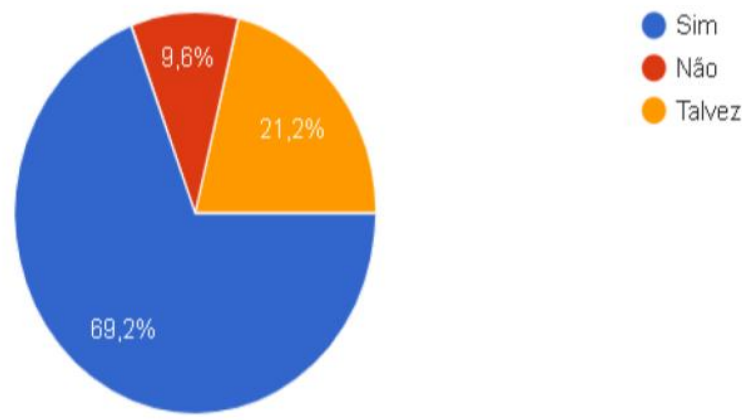

Fonte: Autores (2021).

Do grupo amostral, 26 alunos mostraram-se favoráveis a respostas de que a ferramenta de educação influenciou o seu comportamento como estudante durante a disciplina, conforme demonstrado na figura 6 :

Figura 6 - Influência do WhatsApp para o comportamento estudantil

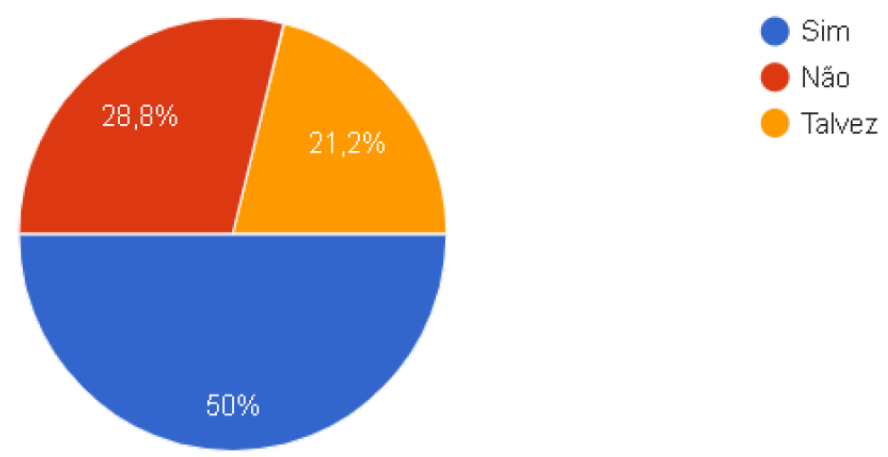

Fonte: Autores (2021).

Os alunos que responderam o questionário $98,1 \%$ afirmou que receberam informações de provas, trabalhos e outros informes pelos grupos criados na ferramenta de educação, em conformidade com a figura 7: 
Figura 7 - Informações recebidas pelos grupos de WhatsApp

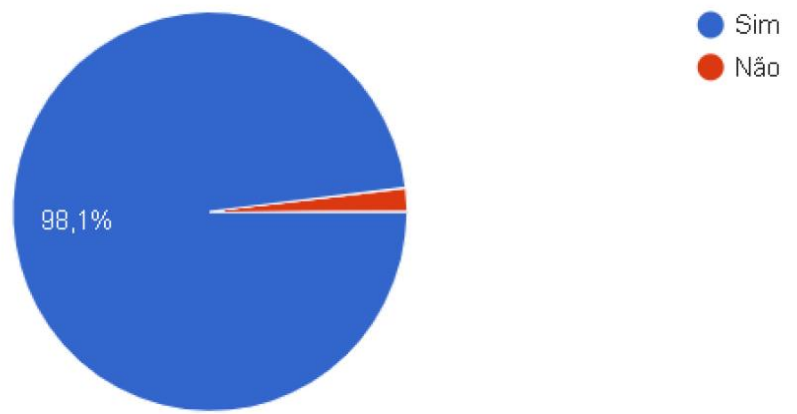

Fonte: Autores (2021).

Segundo a pergunta de número 7, 49 alunos responderam afirmativamente que 0 grupo da disciplina contribuiu para solucionar dúvidas durante o período, atestado na figura 8:

Figura 8 - Solução de dúvidas pelo WhatsApp

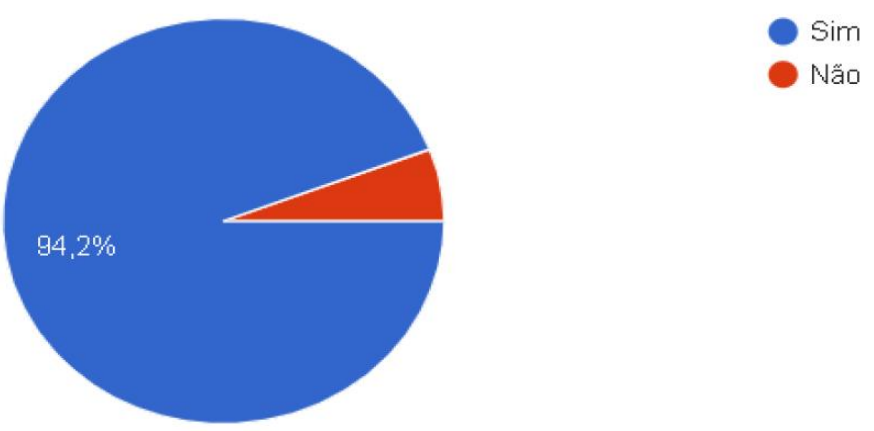

Fonte: Autores (2021).

Segundo os estudantes, apenas 13,5\% disseram que os professores não estavam nesses grupos do WhatsApp, mostrado na figura 9:

\section{Figura 9 - Participação dos professores nos grupos}

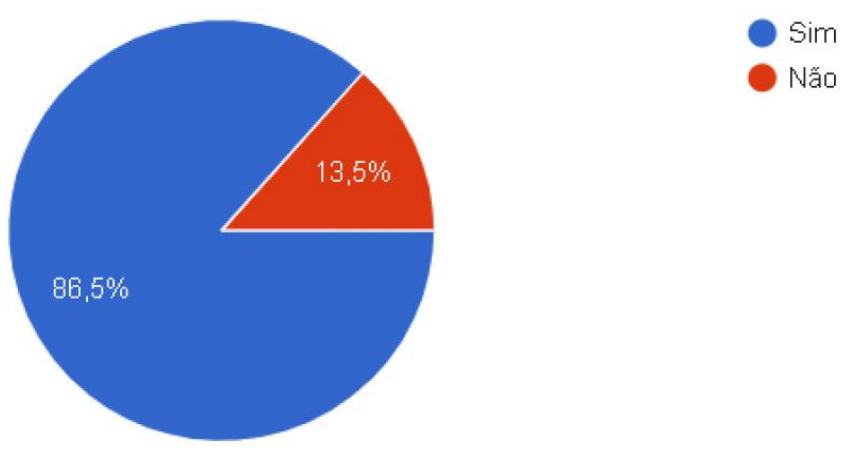

Fonte: Autores (2021).

Em resposta sobre a importância de os professores participarem dos grupos, $42,3 \%$ responderam que é extremamente importante a participação dos mesmos, conforme a figura 10: 
Figura 10 - Importância do professor no grupo de WhatsApp

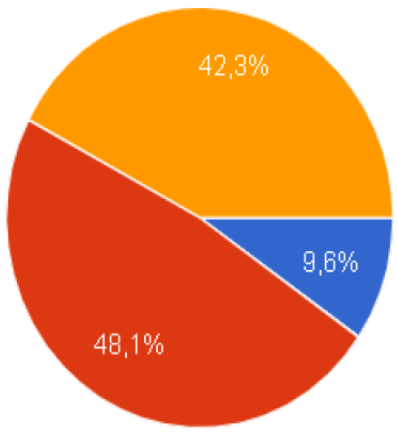

Pouco relevante

Relevante

Extremamente relevante

Fonte: Autores (2021).

Para $80,8 \%$ dos alunos declararam que a participação dos professores no grupo da disciplina, contribuíram para melhorar a relação aluno - professor, como demonstrado na figura 11:

\section{Figura 11 - Melhoria da relação Aluno-Professor}
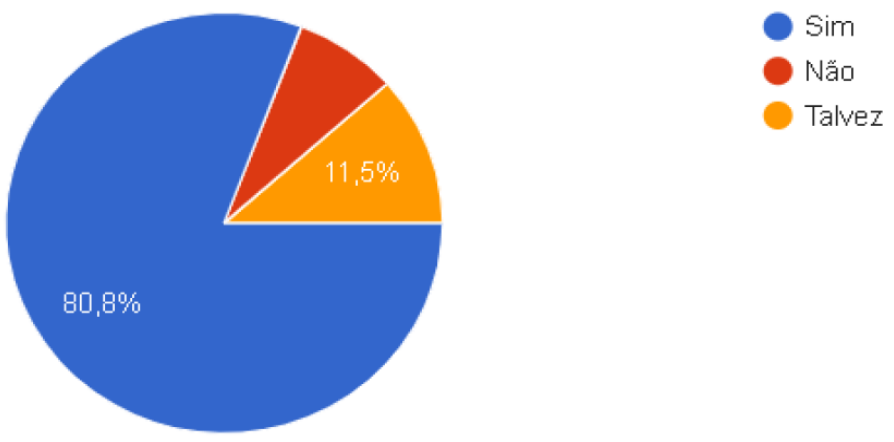

Fonte: Autores (2021).

$\mathrm{Na}$ última pergunta, foi questionado a importância da ferramenta de educação selecionada e foi obtido resposta favoráveis frente o espaço amostral, demonstrado na figura 12:

Figura 12 - Utilização do WhatsApp como ferramenta educacional
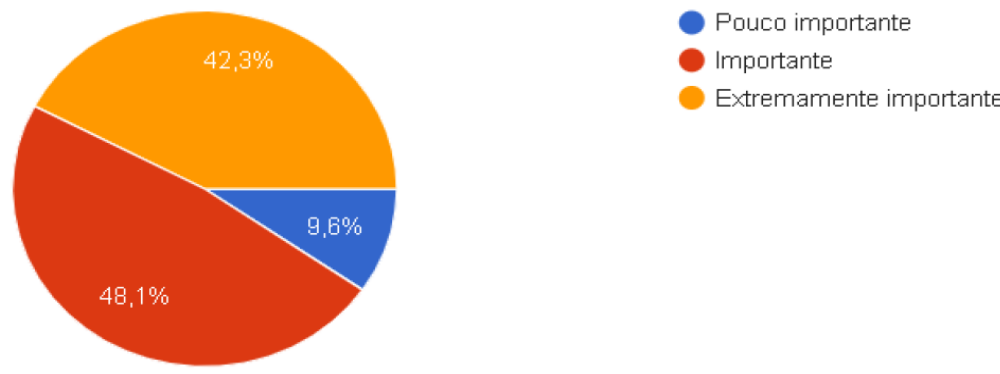

Fonte: Autores (2021).

Visto que as respostas em sua maioria foram de índice afirmativo, conclui-se que as tecnologias geram transformações significativas durante a construção do profissional, essas melhorias são visualizadas na relação crescente entre aluno-professor, na participação efetiva dos alunos durante a oferta da disciplina e nos relatos encontrados durante as respostas do questionário. Tal estudo, viabiliza a importância dos meios de 
comunicação, como o WhatsApp como ferramenta pedagógica, onde o seu uso efetivo carrega melhorias nas partes envolvidas.

\section{CONCLUSÕES}

Este estudo buscou alinhar uma investigação da usabilidade do aplicativo WhatsApp enquanto recurso de apoio as mediações didáticas as disciplinas remotas no curso de engenharia civil e de produção em uma instituição privada de ensino superior. Para tal, buscou-se como universo estudantes dos cursos de engenharia civil de produção do Centro Universitário Anhanguera de Niterói participantes da disciplina de TCC ministrada de modo remoto em virtude da crise sanitária atual. Os resultados demostram de forma significativa o quanto o aplicativo em voga mostrou-se satisfatório ao propósito em questão. Esta constatação reforça a iniciativa deste estudo e levanta também a necessidade de estudos futuros em outras realidades, como em cursos de pósgraduação, cujo perfil dos estudantes tende a ser diferente dos estudantes da graduação.

Como pontos positivos deste estudo destacaram-se por meio das respostas levantadas o dinamismo comunicacional provocado pelo aplicativo, à facilidade de interação contínua, a fluidez do diálogo e da troca de informações, a inserção de materiais diversos para apoio a disciplina em questão, a adesão dos discentes e por fim, a possibilidade de interação também com outras plataformas.

Estes fatores, reforçam a vicissitude do potencial didático do aplicativo WhatsApp e inspiram também a continuidade de experiências diferenciadas que possam ampliar ainda mais sua usabilidade no contexto educacional.

\section{Agradecimentos}

Agradecemos à Fundação Nacional de Desenvolvimento do Ensino Superior Particular (FUNADESP) e ao Centro Universitário Anhanguera de Niterói (UNIAN) pelo auxílio financeiro concedido para a realização do Projeto de Pesquisa que abarca este estudo.

\section{REFERÊNCIAS}

ADEGBIJA, M. V., BOLA, O. O. Perception of Undergraduate a on the adoption of mobile technologies for learning in selected Universities in Kwara State, Nigeria. Procedia Social Behavioral Sciences, 176(1), 352- 356, 2015.

AL-EMRAN, M., ELSHERIF, H. M., \& SHAALAN, K. Investigating Attitudes towards the Use of Mobile Learning in Higher Education. Computers in Human Behavior, 56(1), 93102, 2016.

ALSHRAIDEH, R. S., \& AL-SHRIDA, R. S. Factors affecting the adoption of mobile learning by Jordanian university students: Based on UTAUT model. Current Educational Research, 1(2), 72-80, 2018. 
BRIZ-PONCE, L., JUANES-MENDEZ, J. A., GARCIA-PENALVO, F. J., \& PEREIRA, A. (2016). Effects of Mobile Learning in Medical education: A Counterfactual Evoluation. Journal of Medical Systems, 40(1), 45-57, 2016.

CIRIBELLI, Marilda Corrêa. Como elaborar uma dissertação de Mestrado através da pesquisa científica. Marilda Ciribelli Corrêa, Rio de Janeiro: 7 Letras, 2003.

COSTA, R.D.A., ALMEIDA,C.M.M., LOPES, P.T.C. Aplicativos para dispositivos móveis e metodologias ativas: possibilidades pedagógicas para o ensino e aprendizagem em nível superior. Revista Redin. v. 6 № 1. Outubro, 2017. Disponível em: < https://seer.faccat.br/index.php/redin/article/viewFile/656/512 > Acesso em: 08/03/2020.

DIRETRIZES PARA O CURSO DE ENGENHARIA/ABENGE. Inovação na Educação em Engenharia : Proposta de Diretrizes para o Curso de Engenharia. Brasília, jan. 2018. Disponível em: < http://www.abenge.org.br/file/PropostaDCNABENGEMEI CNI.pdf > Acesso em: 12/03/2020.

FIGUEIREDO, M., GODEJORD, B., \& RODRIGUES, J. The Development of an Interactive Mathematics App for Mobile Learning. INTERNATIONAL CONFERENCE MOBILE LEARNING, 12, 75-81, 2016.

GODOY, M.T.H.; MUNARI, D. B. Análise da produção científica sobre a utilização de atividades grupais no trabalho do enfermeiro no Brasil: 1980 a 2003. Rev. Latino-Am. Enfermagem, Ribeirão Preto, v. 14, n. 5, p. 786-802, 2006.

IMBERNÓN, Francisco. Formação Docente e Profissional: Formar-se para a Mudança e a Incerteza. São Paulo, Cortez, 2002.

KAYE, A. Learning Together Apart. In: KAYE, A. Collaborative Learning Through Computer Conferencing. NATO ASI Series, v. 90, cap. 1, p. 1-24, 1991.

KITZINGER, J. Focus groups with users and providers of health care. In: POPE, C.; MAYS, N. (Org.). Qualitative research in health care. 2. ed. London: BMJ Books, 2000.

MORÉS, A. A universidade e sua função social: os avanços da EaD e suas contribuições nos processos de ensino e aprendizagem. Revista Reflexão e Ação, v.25, n.1, p. 141-159, 2017.

NBR ISO/IEC 9126-1:2003. Tecnologia de informação: Engenharia de software; Qualidade de produto - parte 1: Modelo de qualidade. Rio de Janeiro, jul. 2003.

ROCHA, Fernanda Suzart da; SANTANA, Eloisa Bahia; SILVA, Érica Santos da; CARVALHO, Josiane Silva Martins; CARVALHO, Fernando Luís de Queiroz. Uso de Apps Para a Promoção dos Cuidados à Saúde. III Seminário de Tecnologias Aplicadas em Educação e Saúde.

SACCOL, A., SCHLEMMER, E. \& BARBOSA, J. M-learning e u-learning: novas perspectivas das aprendizagens móvel e ubíqua. São Paulo: Pearson Prentice Hall, 2011. 
SEOW, P. S., \& WONG, S. P. Using a mobile gaming app to enhance accounting education. Journal of Education for Business, 91(8), 434-439, 2016.

VÁZQUEZ-CANO, E. (2014). Mobile Distance Learning With Smartphones and Apps in Higher Education. Educational Sciences Theory \& Pratice v. 14, I.4, p. 1505-1520, 2014.

\title{
THE USABILITY OF THE WHATSAPP APPLICATION AS A RESOURCE OF SUPPORT FOR TEACHING MEDIA TO REMOTE TCC DISCIPLINE IN THE CIVIL ENGINEERING AND PRODUCTION COURSE
}

\begin{abstract}
In the current scenario, with the suspension of face-to-face classes, digital media have become essential. Thus, the present study aims to promote an investigation of the usability of the WhatsApp application as a resource to support didactic mediations in remote disciplines in the course of civil engineering and production at a private higher education institution. It is notorious to note the expansionist use of applications (APPs) as a functional resource to subsidize different services. This functionality was made possible by the progress of cyberspace, associated with the advent of smartphones, which have, among their characteristics, interconnectivity and its easy use. Driven by this panorama, this study sought as a methodological trail to combine quantitative and qualitative research with a descriptive bias. Thus, its results showed how the application in vogue aligns with robust possibilities as a resource to support didactic mediations in the remote disciplines of the courses in question.
\end{abstract}

Keywords: Communication Applications, Collaborative Teaching Practices and Engineering Students. 